\title{
AZO DYES AND HEINZ BODIES
}

\author{
BY \\ PHILIP ROFE \\ From the Industrial Hygiene Research Laboratories, Imperial Chemical Industries Limited, The Frythe, Welwyn, Herts.
}

(RECEIVED FOR PUBLICATION APRIL 13, 1957)

No general hypothesis has been advanced to explain the underlying cause of the appearance of Heinz bodies in erythrocytes. The initial observation of Riess (1882) on a case of potassium chlorate poisoning was followed by that of Heinz (1890) who administered phenylhydrazine to animals. Since then Heinz bodies have been induced by a great variety of substances, principally aromatic and aliphatic nitro-compounds, aromatic amines and organic (notably azo) dyestuffs, a miscellaneous group of organic nitrogen-free compounds (e.g., naphthalene, $2: 4$ dichlorphenol, ascorbic acid), and a number of inorganic substances (e.g., sodium nitrite, sodium sulphite, potassium dichromate, arsine). Heinz bodies are also found normally in cats (Hecht and Wingler, 1952) and their appearance has been observed after splenectomy in animals (Schilling, 1921 ; Freifeld, Schilowa, and Ludwinowsky, 1937) and in human subjects (Zadek and Burg, 1930; Selwyn, 1955). The substance of Heinz bodies has been thought by many to originate from haemoglobin rather than the cell stroma, and recently Beaven and White (1954) have demonstrated that it is spectroscopically similar to insoluble " green haemoglobin" which is described as being "complex, containing haems and other iron-containing pigments bound to denatured globin ". Associated with the appearance of Heinz bodies, though not invariably, are anaemia, methaemoglobinaemia, and verdohaemoglobinaemia: these and other topics have been exhaustively reviewed by Fertman and Fertman (1955).

The use of azo dyes for the colouring of food has led to some preoccupation regarding the possible carcinogenic and toxic effects of the dyes or of the products resulting from reduction-fission at the azo linkage. The current need for more information on dye toxicity is emphasized in the report on colouring matters issued by the Food Standards Committee of the Ministry of Food (1954) wherein reference is made to the work of Hecht and Wingler (1952). These authors point out that although fission products such as aniline, toluidine, or xylidine may not have been proved to be carcinogenic, their other known toxic properties rule out the use of the parent dye in food. A suitable dye might be assessed from this point of view by estimation of the degree of methaemoglobinaemia produced, but Hecht and Wingler (loc.cit.) advocate instead the examination of the blood for Heinz bodies, as this procedure is simpler and more sensitive. These authors reported that no Heinz bodies were produced by azo dyes of the type $\Longrightarrow \mathrm{N}=\mathrm{N}-\mathrm{R}^{(\mathrm{R}=\text { sulphonated aromatic }}$ when the aniline component was sulphonated. This finding accords wiell with the generally accepted view that sulphonation has a detoxicating effect, and enhances the value of the Heinz body test as an indication of the presence of, for example, free amines. In a later paper, Wingler (1953) found that methylation at the para position on the aniline component of benzene-azo-naphthol 6 and $6: 8$ sulphonates likewise removes Heinz body-forming activity.

In the work described in this paper the activity of eight azo dyes (Table 1) has been observed in order to compare the effect of substituent groups in the benzene-azo-naphthalene nucleus. The dyes were administered in the food since an ultimate object has been to assess the significance of Heinz body formation in the problem of food colours for human consumption.

\section{Method}

For each dye tested, 10 male rats weighing 150 to $200 \mathrm{~g}$. were used. Rat cake No. 86* milled to a coarse powder, was mixed with the dye to give a concentration of $2 \mathrm{mM}$. pure dye/100 g. food (equivalent to $0.5 \%$ Sudan yellow); a known proportion of inorganic diluents was present in the dye samples and was allowed for. The daily food intake was limited to $60 \mathrm{~g}$. $/ \mathrm{kg}$. mean body weight in order to avoid the variation which occurs, due to differences in palatability, when larger amounts

*North Eastern Agricultural Cooperative Society Ltd. 
TABLE 1

INDIVIDUAL HEINZ BODY RESPONSES OF RATS FED AZO DYES IN THE DIET

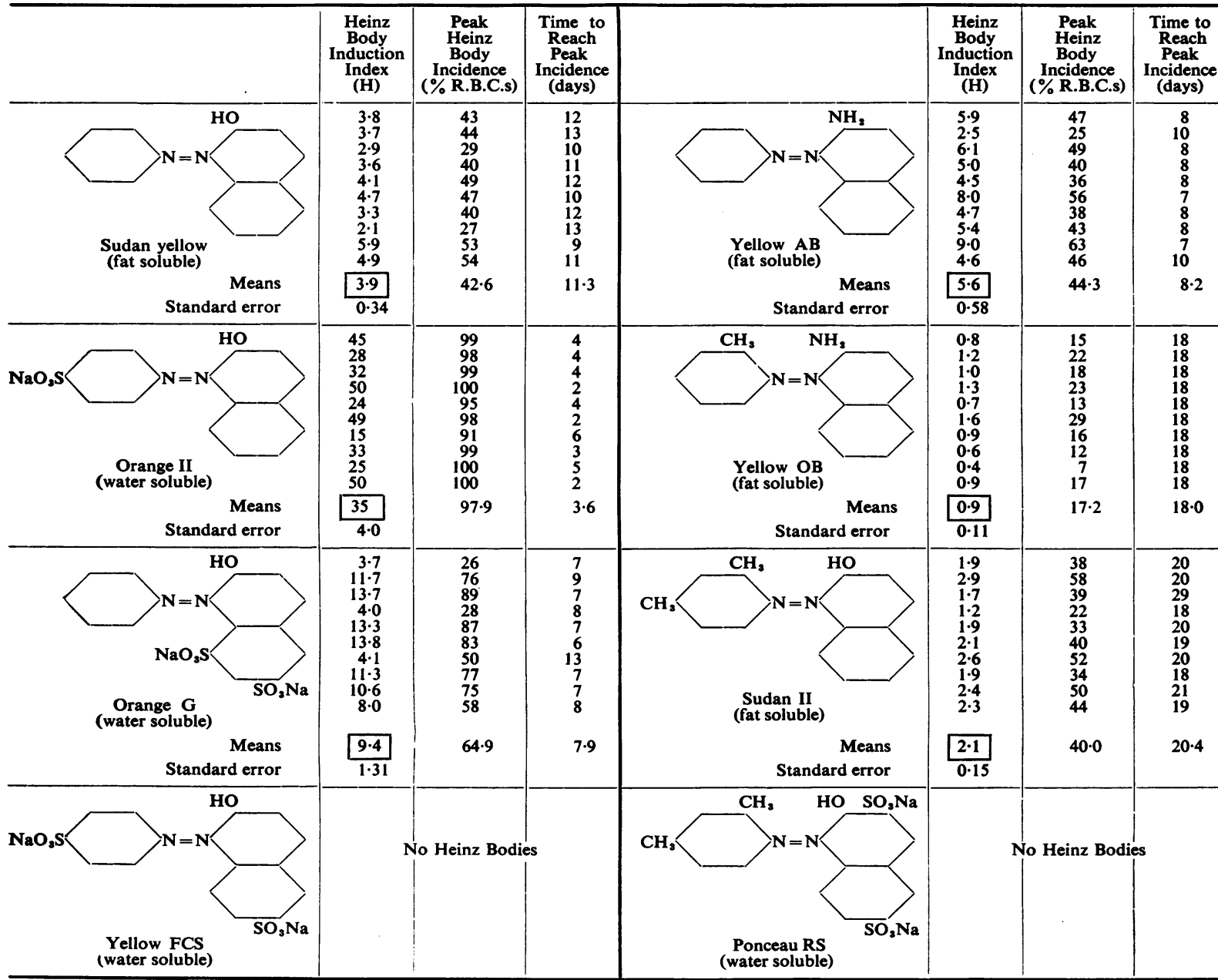

All dyes were fed at a concentration of $2 \mathrm{mM} / 100 \mathrm{~g}$. food, and the total food intake controlled to obtain approximately equimolecular dye ingestion. The figures for Sudan yellow itself are slightly low, as, owing to unpalatability, about $12 \%$ of the dye-food mixture was rejected.

are available to the animals. Spillage of food, which becomes a problem when unpalatable dyes are fed, was avoided by use of specially designed containers which reduced this loss to not more than $0.1 \%$. By these means ingestion of equimolecular quantities of dyes per unit body weight per day were obtained, thus enabling the Heinz body responses to the different dyes to be compared.

Heinz body counts were made at frequent intervals, especially during the period near the peak incidence. To one drop of methyl violet stain (half-saturated solution in normal saline containing $\mathbf{0 . 2} \%$ sodium citrate) on a slide, was added one-quarter this volume of tail blood on the corner of a coverslip: after mixing, the coverslip was released and the edges sealed with "vaseline".
At least 300 red cells were counted on each occasion using $\times 855$ magnification. The greatest care was taken to distinguish Howell-Jolly bodies and reticulocytes, and to ensure that the field under examination was adequately stained to show up the smaller Heinz bodies. Blood from normal rats was not found to contain Heinz bodies.

\section{Results}

The Heinz Body Response.-From day-to-day observation of some 150 rats receiving dyes continuously in the diet, it can be said that the Heinz body level usually takes the following course: there is first a latent period between the beginning of 


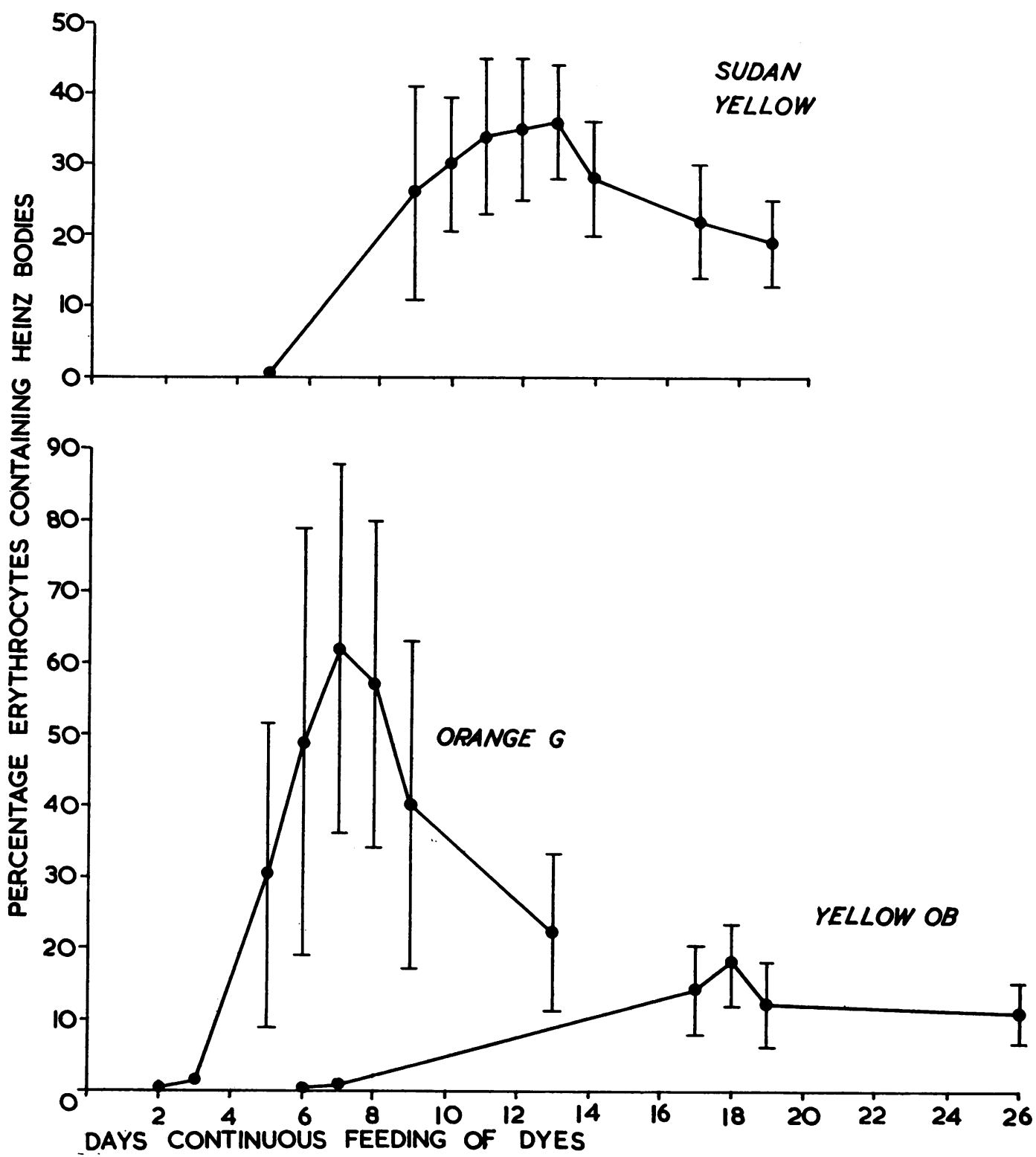

FIG. 1.-The proportion of erythrocytes containing Heinz bodies in groups of 10 rats ingesting in equimolecular quantities either Sudan yellow, orange G, or yellow $O B$. Each point is the mean value; the vertical line represents \pm the standard deviation. These dyes have been chosen to show the large differences in the form of the response, yet the same general pattern is discernible in each case, namely, a latent period, relatively rapid rise to a peak, and a slower fall. Thereafter a fluctuating lower level is maintained so long as the dye is given.

ingestion of dye and the appearance of Heinz bodies in the blood, and this may last one or several days depending on the dye, its concentration, and the individual response of the animal; there follows a relatively rapid rise to a peak frequency of Heinz bodies and this is followed by a fall to a fluctuating lower level which is maintained so long as the dye is consumed. Fig. 1 shows the course of the mean Heinz body level in groups of 10 rats ingesting, in equimolecular quantities, Sudan yellow, orange 
G, or yellow $O B$ (for structures see Table 1).

Comparison of Heinz Body Responses.-In attempting to compare the sizes of the Heinz body responses it is evident that as well as the maximum incidence of Heinz body-bearing erythrocytes attained, the time taken, i.e., the total dose in gram-molecules, must be taken into account. In order to obtain an expression for the true Heinz body-forming potency of one dye relative to that of another, an arbitrary "Heinz body induction index" $(\mathrm{H})$ has been used: this is the highest quotient obtainable by dividing the Heinz body incidence by the time taken to reach it at a stated feeding rate. This value and also the peak Heinz body incidence, with the time taken to reach it, is given for each animal in Table 1. It will be appreciated that the highest quotient is not always obtained from the peak Heinz body incidence: thus an incidence of $80 \%$ on day 4 will give a higher quotient and indicate a higher potency than $85 \%$ on day 5 .

Effect of Sulphonation and of Methylation.-It is seen in Table 1 that p-sulphonation of the aniline component of benzene-azo-2-naphthol (Sudan yellow) to give orange II greatly increases the Heinz body response : the value of $\mathrm{H}$ is raised from 3.9 to 35. Disulphonation $(6: 8)$ of the naphthol component instead, to give orange $G$, also increases the response, in this case to 9.4. But monosulphonation of both aniline and naphthol components gives yellow FCS, which is completely ineffective as a Heinz body inducer. The results for Sudan II and Ponceau RS show that unilateral sulphonation is sufficient to abolish the Heinz body-inducing property if the contralateral component relative to the azo link is dimethylated.

Wingler (1953), using cats, found ortho- or paramethylation to abolish activity in the water-soluble benzene azo-2-naphthol- 6 and $6: 8$ sulphonates. The effect of methylation of the benzene component of benzene-azo-2-naphthol (Sudan yellow) is seen (Table 1) in the results for Sudan II, and of methylation of benzene-azo-2-naphthylamine (yellow AB) is seen in the figures for yellow OB. In the former case methylation greatly delays the attainment of the peak value, which is not altered, and reduces the index $\mathrm{H}$ from 3.9 to 2.1 : application of the $t$ test to this result shows a significant difference $(P<0.005)$. In the latter case, there is both delay and a smaller peak: $\mathrm{H}$ falls from 5.6 to 0.9 . These findings are the more valid since solubility complications are excluded, all four dyes being fat soluble and water insoluble.

The effect of p-sulphonation or of p-methylation of aniline on the Heinz body-producing activity of this substance has been observer in a few animals in the course of the present work. This is of interest since aniline or a homologue of aniline will be an expected primary metabolite resulting from reductive fission at the azo link. It was found that the mean peak Heinz body response to feeding $0.1 \%$ aniline in the diet was $24 \%$ in six days, but when sulphanilic acid, at four times the molecular concentration of $0.1 \%$ aniline, was given in the diet no Heinz bodies were seen. On the other hand, p-toluidine at a molecular concentration equal to that of $0.1 \%$ aniline produced a peak response of $48 \%$ after 15 days: this result contrasts with Wingler's finding (1953) that only the m-isomer causes Heinz bodies; however, he used a much smaller dose injected daily in cats for only $\mathbf{1 0}$ days. Daniel (1956) identified aniline in the urine of rabbits given Sudan yellow $(0.5 \mathrm{~g} . / \mathrm{kg}$.) although $85 \%$ of the material in the urine (which was $51 \%$ of the dose administered) could be accounted for as p-aminophenol and 1-amino-2-naphthol. The formation of Heinz bodies in rats, however, is not due to p-aminophenol, for neither feeding at twice the molecular concentration of $0.1 \%$ aniline for 23 days, nor subcutaneous injection at $240 \mathrm{mg}$. $/ \mathrm{kg}$. per day for six days caused them to appear.

\section{Discussion}

Evidence against Heinz Body Formation by Unchanged Dye.-Heinz bodies are formed only in erythrocytes and are not found in immature precursors. The question arises as to whether the active agents in the plasma are the dyes or their products resulting from reductive fission at the azo link. While a direct answer cannot be given, some incidental observations bearing on this have been made which suggest that the dyes themselves are not responsible. The fat-soluble dyes referred to in Table 1 cause intense coloration of the plasma, whereas the plasma of rats receiving the watersoluble dyes, with the possible exception of orange II, are strikingly free from colour: this also applies to the water-soluble dye azo geranine* which leads to vigorous Heinz body formation. If the dyes as such were the effective agents, it would be expected that those which are not seen in the plasma would be relatively inactive, but, in fact, Table 1 shows that the two water-soluble dyes quoted give much higher values for $\mathrm{H}$ (35 and 9.4) than the fat-soluble dyes $(5 \cdot 6,3 \cdot 9,2 \cdot 1$, and $1 \cdot 0)$. On the other hand, where a dye is found in the plasma it would be expected to be found in the blood cell, since this is the site of Heinz body formation, but, in fact, the dye appears to be excluded from the red cell, for ether extracts

*Disodium salt of 8-acetamido-2-benzeneazo-1-naphthol-3:6 disulphonic acid. 
of packed erythrocytes from rats fed the fat-soluble Sudan yellow or yellow AB show no colour, although these dyes produce particularly intense plasma coloration.

Heinz Body Test and Carcinogenicity.-Wingler (1953), referring to the possible carcinogenicity of dyestuffs, has stated that the failure of a dye to produce Heinz bodies may be taken as proof that no physiologically active fission products have arisen in the blood. While dubious carcinogens such as aniline and toluidine are certainly Heinz body formers, the same cannot be said for $\beta$-naphthylamine which, although a potent carcinogen, has been found under the conditions of the present study to have no Heinz body-producing activity. Wingler compares the loss of carcinogenicity which occurs when dimethylamino-azo-benzene is methylated at the p-benzene position with the loss of Heinz bodyforming activity of azo dyes by methylation, but feels that no direct relation between a positive Heinz body test and carcinogenic effect exists. This view is confirmed by the following evidence: Frodsham (1956) fed azo geranine or red $10 \mathrm{BS}^{*}$ in the diet at a concentration of $1.5 \%$ each dye, to 20 rats, for a period of two years without finding tumours; Allmark, Grice, and Lu (1955) after feeding yellow AB or yellow OB $(0.3 \%)$ in the diet, each to a total of 50 rats for 65 weeks, found only two neoplasms in the former case and none in the latter. But in the course of the present work all these dyes have been found to produce Heinz bodies, particularly the former two, which are very active.

Changes Concurrent with Presence of Heinz Bodies.-Irrespective of the value of the Heinz body test as a very sensitive indicator of the presence in the blood of certain toxic amines and of many other substances, the question remains as to whether the presence of Heinz bodies as such has any adverse effect on the animal. Rats eating about 1 to $1.5 \mathrm{~g} . / \mathrm{kg}$./day azo geranine for 75 days showed no external symptoms apart from a slight cyanotic appearance despite a mean peak Heinz body level of $80 \%$ falling to a maintained level of $30 \%$. Internal changes included a moderate though wellcontrolled anaemia, pronounced reticulocytosis, and splenomegaly: the anaemia and reticulocytosis were presumably due to the destruction of affected erythrocytes by the spleen. The maintenance of a Heinz body level as a result of regular ingestion of an otherwise harmless substance must therefore lead to a state of chronic hyperactivity of the reticuloendothelial and haemopoietic systems. In the

*Disodium salt of 8-amino-2-phenylazo-1-naphthol-3 : 6-disulphonic acid. rats, a return to ordinary diet resulted in a return to normal values within two weeks, apart from a slight compensatory polycythaemia which remained for months; but whether irreversible changes would not occur if such a substance were taken during a much longer period is not known. Further data on these topics will be published.

\section{Summary}

1. The induction of Heinz bodies in rats by azo dyes has been studied in relation to the effect of different substituent groups attached to the benzeneazo-naphthalene nucleus. Eight dyes having this nucleus in common were fed continuously at equimolecular rates by admixture in the diet, each dye to a group of 10 rats, and the blood examined at intervals for Heinz bodies.

2. Although the magnitude and timing of the Heinz body response to different dyes varied greatly, the same basic pattern was observed in each case: a latent period, then a relatively rapid rise to a peak concentration of affected erythrocytes, followed by a slower fall to a fluctuating lower level which was maintained so long as the dye was given.

3. An arbitrary "Heinz body induction index" has been used to facilitate the comparison of the true potencies of the dyes irrespective of their different rates of accumulation.

4. It was found that sulphonation of the benzeneazo-naphthol molecule in either component increased Heinz body induction, but sulphonation of both components abolished it. Methylation of the phenyl group in benzene-azo-naphthol and in benzene-azo-naphthylamine decreased Heinz body induction. Complete loss of activity was obtained by methylation of the phenyl component and sulphonation of the naphthol group in addition in benzene-azo-naphthol. Sulphonation was also found to remove the Heinz body-producing activity of aniline, which is an expected dye metabolite.

5. The coloration of the plasma by ingested fatsoluble dyes but not by water-soluble dyes shows no correlation with Heinz body-producing activity and suggests, along with other evidence, that dye metabolites rather than the dyes as such are the effective agents.

6. With regard to carcinogenicity of dyestuffs and Wingler's (1953) proposal that an absence of Heinz bodies showed an absence of physiologically active amines, the carcinogenic amine $\beta$-naphthylamine was not found to cause Heinz bodies. Four noncarcinogenic dyes were shown to cause Heinz bodies.

I wish to acknowledge the support and encouragement of Dr. M. W. Goldblatt, C.B.E., throughout this work, and his kindness in undertaking translation from the 
German and criticism of the manuscript. I am also indebted to Mr. D. W. Swanston for valued technical assistance.

\section{REFERENCES}

Allmark, M. G., Grice, H. C., and Lu, F. C. (1955). J. Pharm. Pharmacol., 7,591 .

Beaven, G. H., and White, J. C. (1954). Nature (Lond.), 173, 389.

Danee, J. W., (1956). Biochem. J. 64, 1P.
Fertman, M. H., and Fertman, M. B. (1955). Medicine (Baltimore), 34, 131 .
Freifeld, F., Schilowa, A., and Ludwinowsky, R. (1937). Folia haemat. (Lpz.), 56,333 .

Frodsham, J. (1956). Personal communication.

Hecht, G., and Wingler, A. (1952). Arzneimittel-Forschung, 2, 192 Heinz, R.' (1890). Virchows Arch. path. Anat., 122, 112. Quoted by Fertman and Fertman (1955).

Ministry of Food (1954). Food Standards Committee Report on Colouring Matters, H.M. Stationery Office, London.

Riess, (1882). Berl. klin.Wschr. 19, 785. Quoted by Fertman

Schilling, V. (1921). Dtsch. med. Wschr., 47, 825. Quoted by Webster, S. H. (1949), Blood, 4, 485 .

Selwyn, J. G. (1955). Brit. J. Haemat., $1,173$.

Selwyn, J. G. (1955). Brit. J. Haemat., 1, 173

Zadek, I., and Burg, K. (1930). Folia haemat. (Lpz.), 41, 333.

\section{THE JULY (1957) ISSUE}

The July (1957) issue contains the following papers:-

Assessing the Health of the Industrial Worker. By R. S. F. Schilling.

The Control of Industrial Bladder Tumours. By T. S. Scott and M. H. C. Williams.

A Routine Method for the Preparation of the Cells of Urine. By Philip Rofe.

The Testing of Life-jackets. By R. R. Macintosh and E. A. Pask.

A Study of the Thermal Environment in Non-ferrous Foundries. By D. Turner.

Gamma Radiography in Industry. By J. P. W. Hughes and O. Shaw.

The Corrosion of Metal by Palmar Sweat. By K. J. Collins.

Excretion of Urinary Coproporphyrin in Lead Poisoning. Parts 1 and 2. By Václav Holeček.

Tracheotomy in Major Chest Injuries. By S. A. Gueukdjian.

Miscellanea:

A House-Surgeon's Observations on Bronchitis in North Stafiordshire Pottery Workers in 1864. By A. Meiklejohn.

"A Study of Attitudes to Factory Work": A Review. By H. G. Maule.

The British Occupational Hygiene Society.

Book Reviews.

A number of copies are still available and may be obtained from the Publishing Manager, British Medical Association, Tavistock Square, W.C.1, price 12s. 6d. 